\title{
Herlyn-Werner-Wunderlich Syndrome: A Case Report
}

\section{S M Saiful Islam ${ }^{1}$}

${ }^{1}$ Department of Paediatrics and Paediatric Cardiology Imperial Hospital

Chattogram, Bangladesh.
*Correspondence to:

\section{Dr. S M Saiful Islam}

Consultant

Imperial Hospital

Chattogram, Bangladesh.

Mobile : +8801646662992

Email:smsaifuli8@gmail.com

Date of Submission : $\quad 15.05 .2019$

Date of Acceptance : $\quad 30.06 .2019$

www.banglajol.info/index.php/CMOSHMCJ

\begin{abstract}
Herlyn-Werner-Wunderlich Syndrome (HWWS) is a rare developmental disorder of female urogenital tract consisting of paired uterus, obstructed hemivagina and unilateral renal agenesis. Here, authors described a case of a 10 years old girl with HWWS who presented to pediatric emergency with recurrent lower abdominal pain shortly following menstruation and dysmenorrhea. The diagnosis was confirmed by abdominal MRI. Examination under anesthesia revealed two vaginal opening, of which left is widely open and the right is completely closed with bulging hymen. A hymeneal incision was made with evacuation of the cavity. At present, the preferred surgical option is resection of as completely of the obstructing vaginal septum as possible and marsupialization for patients with completely obstructed hemivagina with blind hemivagina and incompletely obstructed hemivagina. The outcome of patients with HWWS is generally good with early detection of illness and prompt management. They can have normal sexual relations with preserved fertility.
\end{abstract}

Key words : Herlyn-Werner-Wunderlich Syndrome; Paired uterus; Hemivagina.

\section{INTRODUCTION}

Herlyn-Werner-Wunderlich Syndrome is a rare congenital abnormality of Müllerian duct characterized by triad of uterine didelphys, blind hemivagina and unilateral renal agenesis. This disorder was first reported as Herlyn-Warner syndrome (Renal agenesis and unilateral blind hemivagina) in 1971 by Herlyn-Warner ${ }^{1}$. In 1976, Wunderlich described the triad of ipsilateral renal agenesis, bicornuate uterus and simple vagina with isolated hematocervix ${ }^{2}$. The exact incidence of this defect is not known but it is estimated between $0.1 \%-3.8 \%$ among women ${ }^{3-7}$. Most of the patients present at peripubertal age with progressively worsening lower abdominal pain, gradually increasing pelvic mass and dysmenorrhea. The syndrome can rarely present at early childhood or neonatal period ${ }^{8,9}$.

Here, we described the case of a 10 years old girl who presented to pediatric emergency with recurrent lower abdominal pain shortly following menstruation and dysmenorrhea associated with didelphys uterus, ipsilateral blind hemivagina and unilateral renal agenesis.

\section{CASE REPORT}

A 10 years old previously healthy girl presented to pediatric emergency with two months history of gradually progressive, episodic right lower abdominal pain radiating to right thigh. Pain was affecting her daily life. She had previously been seen twice at the same Department. Blood workup were normal but neither imaging studies nor gynecological examination were done at that time. She was being treated for a presumptive referred hip pain with analgesics. Menarche had occurred three months earlier associated with dysmenorrhea, periods were regular and lasted about seven days. Pain progressively worsens with each menses. She achieved her puberty at the age of nine years. Her development was normal. 
On presentation, she was hemodynamically normal and afebrile, in no pain, with a tender, movable right pelvic mass extending to right suprapubic area. Pubertal development showed normal. External genital examination revealed tender, bulging imperforate hymen on the right side, while the left hemivagina was patent and opened in the vestibule.

Laboratory exams were normal and a normal urine routine examination results. An ultrasound abdomen showed absence of right kidney and a large cystic mass measuring about $11.2 \times 7$ $\mathrm{cm}$, arising from pelvis extending to right side of lower abdomen in close proximity of right ovary. An abdominal MRI detected agenesis of the right kidney, didelphys uterus communicating with double vagina, of which the right vagina was obstructed. Consequently, there was hematocolpos within the right uterine cavity and to lesser extent in the right fallopian tube causing hematocolpommetria and hematosalphinx. Ovaries were normal with follicle on each side.

The girl was admitted for Examination Under Anesthesia (EUA) which revealed two vaginal opening, of which left is widely open and the right is completely closed with bulging hymen. A hymeneal cruciate incision was done with evacuation of about $300 \mathrm{ml}$ of clotted blood. Further repair was planned in the future. She was discharged on 2 nd day with no further complication.

\section{DISCUSSION}

HWWS is a rare congenital defect of mullerian (Paramesonephric) ducts described as triad of paired uterus, obstructed hemivagina and unilateral agenesis of kidney. The female urogenital organs develop from the Wolffian (Mesonephric) and the Mullerian ducts. The Mullerian ducts unite together in the midline and give rise to uterine tubes, uterus and upper two-thirds of vagina. The lower third of the vagina derives from urogenital sinus. Non-development or abnormal development of Mullerian ducts include: i) Agenesis or hypoplasia ii) Defects of vertical fusion iii) Anomaly of the lateral fusion, which can cause agenesis or hypoplasia of uterus, didelphys uterus, unicornuate or bicornuate uterus and agenesis or duplication of vagina. HerlynWerner-Wunderlich Syndrome results from non-development or vertical and lateral non-fusion of Mullerian ducts between 6-9 weeks of intrauterine life. The anomalous differentiation of Wolffian and Mullerian ducts can also cause abnormalities of kidneys with renal agenesis being the commonest ${ }^{7-14}$.

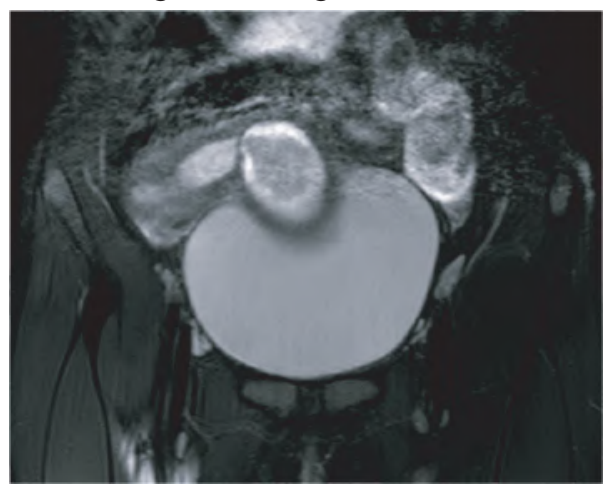

Figure 1 : T2-Weighted image SPAIR shows two uterine cavities (Arrows) filled with hyperintense fluid and hematocolpos (Star). Bilateral normal ovaries are present (Not shown)

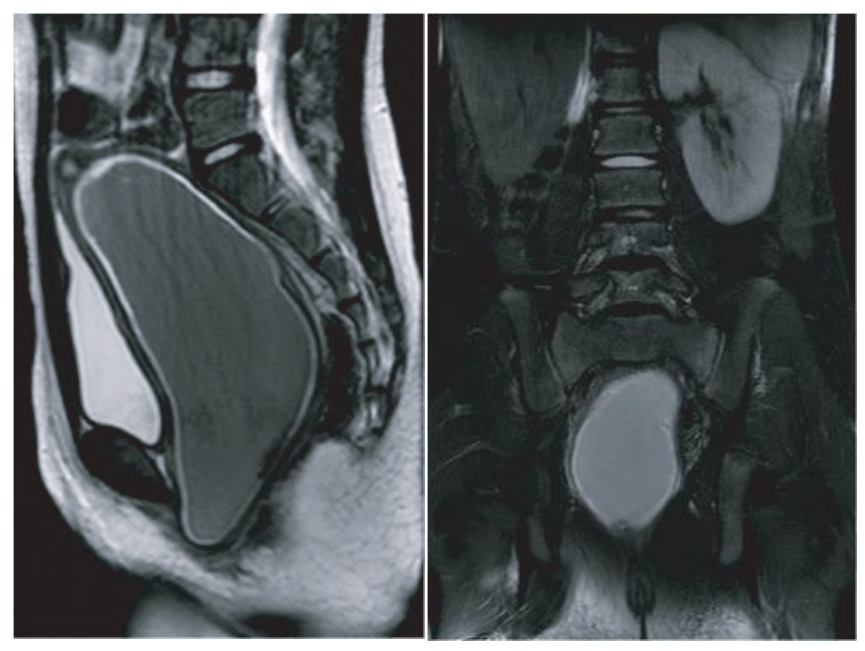

Figure 2 : Sagittal T2 weighted image and coronal T2 SPAIR image show hematocolpos (Star) blood products residual (Arrow) and single left kidney.

Patients with HWWS can be divided into two groups ${ }^{3}$.

i) Completely obstructed hemivagina

- With blind hemivagina

The hemivagina is entirely blind. The uterus behind the septum is totally separated from the opposite uterus and there is no connection between two identical uterus and vagina.

- Cervicovaginal atresia without communicating uteri The hemivagina is entirely obstructed and the cervix behind the septum is atretic.

ii) Incompletely obstructed hemivagina

- Partial reabsorption of the vaginal septum

The hemivagina is partially obstructed with a tiny connection between two vaginas. The uterus behind the septum is entirely separated from the opposite uterus.

- With communicating uteri

The hemivagina is totally blocked with a tiny connection between the two cervices.

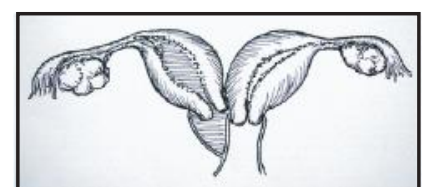

Figure 3a : Completely obstructed hemivagina with blind hemivagina with hemivagina cervicovaginal atresia uteri

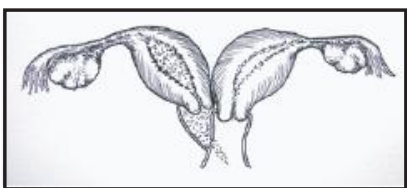

Figure 4a :Incompletely obstructed hemivagina partial reabsorption of the vaginal septum

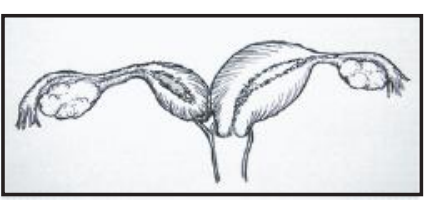

Figure $3 \mathbf{b}$ : Completely obstructed without communicating

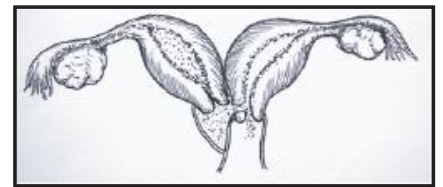

Figure 4b : Incompletely obstructed hemivagina with with communicating uteri 
Patients with HWWS usually present in peripubertal age with nonspecific symptoms. The diagnosis is frequently delayed because of the rarity of disorder. Females with completely obstructed hemivagina present earlier than those with incompletely obstructed hemivagina.

Duration between menarche and onset of symptoms is much shorter in patients with complete obstruction than those with incomplete obstruction ${ }^{3}$. Patients commonly present with progressively increasing, cyclic lower abdominal pain or dysmenorrhea, pelvic mass, fever and vomiting; usually associated with each menstruation. Women with partially obstructed hemivagina may complain of foul smelly purulent or bloody vaginal discharge secondary to pyocolpos. There is a right sided preponderance of the disorder. Clinical examination reveals tender, mobile pelvic mass ${ }^{3,14-17}$.

HWWS may mimic with acute appendicitis, twisted right ovarian cyst, ectopic pregnancy, pelvic inflammatory disease and right pelvic tumors.

Blood investigations are usually unremarkable. Ultrasound (US) and MRI abdomen are very useful, noninvasive diagnostic tools of choice. US helps to establish the diagnosis by detecting uterovaginal duplication, the absence of the ipsilateral kidney with compensatory hypertrophy of contralateral kidney and hematocolpos or hematometrocolpos. 3D US has diagnostic superiority. MRI is extremely helpful in accurately delineating the uterovaginal anatomy, the communication between vaginal or uterovaginal lumen, the nature of the fluid content with other associated complications like endometriosis, pelvic inflammations and adhesions. In addition, MRI plays an important role in classifying the complex Mullarian duct anomalies and finally, in deciding the suitable surgical options. Hysterosalpingography reveals contrast media flowing through the uterovaginal communication to contralateral uterus and then the obstructed hemivagina ${ }^{18-21}$.
Though, laparoscopic examination has been mentioned in some literature, it is not routinely used ${ }^{4,6}$.

Complications like hematocolpos can develop shortly after menarche. Hematometra, hematosalpinx with bleeding in the periadnexal and peritoneal space may develop in severely affected women secondary to retrograde menstrual flow. When diagnosis is delayed, it may cause endometriosis, pyosalpinx or even pyocolpos. If not appropriately intervened in time, the disease may progress to secondary endometriosis, pelvic adhesions which can lead to infertility 22,23 .

At present, the preferred surgical option is resection of as completely of the obstructing vaginal septum as possible and marsupialization for patients with completely obstructed hemivagina with blind hemivagina and incompletely obstructed hemivagina. This promptly alleviate symptoms and avoidcomplications with preservation of reproductive function. Ipsilateral hysterectomy is recommended for female with completely obstructed hemivagina with cervicovaginal atresia $3,4,6,8,23-24$.

\section{CONCLUSION}

Though, HWWS is a rare disorder the outcome is generally good with early detection of illness and prompt management ${ }^{9}$. So young patient with dysmenorrhaea should be evaluated with USG or MRI. They can have normal sexual relations with preserved fertility. The rate of spontaneous miscarriage is high (40\%). Renal agenesis needs lifelong follow up.

\section{DISCLOSURE}

All the authors declared no competing interest. 


\section{REFERENCES}

1. Herlyn U, Werner H. Simultaneous occurrence of an open Gartner duct cyst, a homolateral aplasia of the kidney and a double uterus as atypical syndrome of abnormalities. GeburtshilfeFrauenheilkd. 1971;31:340-347.

2. Wunderlich M. Unusual form of genital malformation with aplasia of the right kidney. Zentralbl Gynakol. 1976;98:559e62.

3. Zhu L, Chen N, Tong JL. New Classification of Herlyn-Werner-Wunderlich Syndrome. Chin Med J. 2015;128:222-2225.

4. Zurawin RK, Ietrich JE, Heard MJ et al. Didelphic uterus and obstructed hemivagina with renal agenesis: case report and review of the literature. J Pediatr, Adolesc Gynecol. 2004 ;17 : 137-141.

5. Burgis J. Obstructive Müllerian anomalies: Case report, diagnosis, and management. Am J ObstetGynecol. 2001;185:338-344.

6. Aveiro AC, Miranda AJ, Cabral et al. Herlyn-Werner-Wunderlich syndrome: A rare cause of pelvicpain in adolescent girls. BMJ Case Reports. 2011.

7. Wu TH, Wu TT, Ng YY. Herlyn-Werner-Wunderlich Syndrome Consisting of Uterine Didelphys, Obstructed Hemivaginaand, Ipsilateral Renal Agenesis in a Newborn. Pediatrics and Neonatology. 2012;53:68-71.

8. Angotti R, Molinaro F, Bulotta AL et al. Herlyn-Werner-Wunderlich syndrome: An "early" onset case report and review of Literature; International Journal of Surgery Case Reports. 2015;11:59-63.

9. Orazi C, Lucchetti MC, Schingo PM et al. Werner-Wunderlich syndrome: uterus didelphys, blind hemivaginaand ipsilateral renal agenesis. Sonographic and MR findings in 11 cases. PediatrRadiol. 2007; 37: 657-665.

10. Acien P. Embrological observations on the female genital tract. Hum Reprod. 1992; 7: 437-445.

11. American Fertility Society. The American Fertility Society classifications of adnexal adhesions, distal tubal occlusion, tubal occlusion secondary to tubal ligation, tubal pregnancies. Mullerian duct anomalies and intrauterine adhesions. FertilSteril. 1988; 49: 944-955.

12. Sadlar TW, Langman J (eds). Langman's Medical Embryology, $8^{\text {th }}$ edn. Philadelphia: Lippincott Williams and Wilkins. 2000

13. Arkan T, Harma M, Harma MB et al. Herlyn-Werner-Wunderlich syndrome (Uterus didelphys, blind hemivagina and ipsilateral renal agenesis) - a case report, J Turkish-German GynecolAssoc. 2010; 11: 107-109

14. Wang J, Zhu L, Lang J. Clinical characteristics and treatment of Herlyn-Werner-Wunderlich syndrome. Arc.ynecol.Obstet. 2014;290(5):947950.

15. Fedele L, Motta F, Frontino GE. Double uterus with obstructed hemivagina and ipsilateral renal agenesis: pelvic anatomic variants in 87 cases, Hum. Reprod. 2013;37(7):657-665.

16. Zhu L, Lang J, Tong J. Clinical characteristics of 70 patients with Herlyn-Werner-Wunderlich syndrome. Int. J. Gynaecol. Obstet. 2013;121(2):173-175.

17. Cinzia OM, Lucchetti C, Schingo PMS et al. Herlyn-Werner-Wunderlich syndrome uterus didelphys, blind hemivagina and ipsilateral renal agenesis. Sonographic and MR findings in 11 cases, Pediatr. Radiol. 2007;37(7):657-665.

18. Yavuz A, Bora A, Kurdo GM et al. Werner-Wunderlich syndrome merits of sonographic and magnetic resonance imaging for accurate diagnosis and patient management in 13 cases, Pediatr. Adolesc. Gynecol. 2014

19. Angthong W, Visetsripong M, Amornvithayachan O. Herlyn-Werner-Wunderlich syndrome emphasizes ultrasonographic and MR findings: A Case report and review of the literatures. J Med Assoc Thai. 2012;95(Suppl 12):S129-S133.

20. Vescovo RD, Battisti S, Paola VD et al. Herlyn-werner-wunderlich syndrome: MRI findings, radiological guide (Two cases and literature review), and differential diagnosis. BMC Medical Imaging. 2012;12:4

21. Tong J, Zhu L, Chen N, Lang J. Endometriosis in association with Herlyn Werner Wunderlich syndrome. FertilSteril. 2014; 102:790-794.

22. Gholoum S, Puligandla PS, Hui T. Management and outcome of patients with combined vaginal septum,bifid uterus and ipsilateral renal agenesis (Herlyn Werner Wunderlich syndrome). J PediatrSurg. 2006;41:987-992.

23. Candiani GB, Fedele L, Candiani M. Double uterus, blind hemivagina and ipsilateral renal agenesis: 36 cases and long-term follow-up. ObstetGynecol. 1997; 90: 26-32.

24. Propst AM, Hill JA Anatomic factors associated with recurrent pregnancy loss. Semin. Reprod. Med. 2000;18:341-350 